\title{
Multi-channel Detector Module for Multi-energy Gamma Ray Computed Tomography
}

\author{
André Bieberle $^{1}$ (I) $\cdot$ Ronny Berger $^{1} \cdot$ Philipp Stave $^{1} \cdot$ Uwe Hampel $^{1,2}$ (i)
}

Received: 14 April 2021 / Revised: 5 August 2021 / Accepted: 13 August 2021 / Published online: 31 January 2022

(C) The Author(s) 2022, corrected publication 2022

\begin{abstract}
In this paper an enhanced signal processing electronics for an existing multi-channel detector module for gamma ray computed tomography is presented. The detector electronics is able to evaluate gamma photon energies by measuring pulse duration times, which makes it perfectly suitable for attenuation measurements with multi-energy and/or multiple isotopic sources. The duration time of each voltage pulse generated by a gamma photon within the radiation detector is measured using a complex programmable logic device. A sophisticated logic circuit for eight detector channels is designed to acquire the pulse duration time spectra in a total of 256 channels per detector channel in parallel. This paper introduces the basic concept, describes the general and a specific CPLD design, provides an analysis of the accuracy and presents measured pulse duration time spectra.
\end{abstract}

Keywords Gamma ray computed tomography $\cdot$ Radiation detection $\cdot$ Complex programmable logic device

\section{Introduction}

Computed tomography (CT) is widely used in medicine and non-destructive testing. However, it has also found some application in multiphase flow studies, e.g. for chemical reactors [1], silo discharging [2], centrifugal pumps [3], sandwich packings [4], vertical pipes with defined flow constrictions [5], spout bed reactors [6] and novel chemical reactor designs [7]. Because the latter typically deals with metallic vessels of considerable radiation attenuation, the use of gamma ray computed tomography with high energetic isotopic sources has advantages versus X-ray tomography. Already in 1980, de Vuono et al. [8] presented a design of an isotopic CT scanner for two phase flow measurements. It consisted of 48 collimated scintillator detectors made of $\mathrm{NaI}$ crystals coupled to photo multipliers. The detectors are arranged in an arc shape with a ${ }^{137} \mathrm{Cs}$ source of $18.2 \mathrm{mCi}$

André Bieberle

a.bieberle@hzdr.de

1 Institute of Fluid Dynamics, Helmholtz-Zentrum DresdenRossendorf, Bautzner Landstraße 400, 01328 Dresden, Germany

2 Chair of Imaging Techniques in Energy and Process Engineering, Technische Universität Dresden, 01062 Dresden, Germany activity in its focal point. The whole arrangement could be rotated for obtaining radiographic projections from different angular positions. Scanning of two-phase flow in a pipe of approx. $150 \mathrm{~mm}$ diameter was demonstrated. Subsequently, similar scanners have been developed by other groups, e.g. [9-13]. In 1997, Johansen et al. [14] presented a dual sensor flow imaging tomographic system for the investigation of three-phase flow, i.e. gas, water and oil. It comprises an eight-electrode capacitance tomography system together with a gamma ray tomography system using ${ }^{241} \mathrm{Am}$ as isotopic source, which simultaneously obtained electrical and radiation data from the flow in a pipe section with an inner diameter of $80 \mathrm{~mm}$. Temporal resolution of this system was given as 30 frames per second.

In 2008, our group introduced a high resolution gamma ray computed tomography (HireCT) scanner that comprises a ${ }^{137}$ Cs source and a scintillation detector arc with 320 detector pixels in total [9]. The detectors are operated in pulse count mode and each detector channel contains an energy discrimination stage to exclude scattered gamma photons from the measurement. Thus, very good linear attenuation measurements over up to ten half-widths could be demonstrated. Additionally, in 2010 an improved thermal design of the detector arc was presented, which was carefully developed to be able to provide a high measurement reproducibility at varying temperature and humidity [15]. Based on this, the HireCT scanner was successfully applied 
for investigations of gas-liquid flows, e.g. in an electrically heated rod bundle [16], novel chemical reactor prototypes [7, $17,18]$ and hydraulic couplings [19, 20]. In 2013, our group presented a smaller but more compact gamma ray CT scanner (CompaCT). The electronic and thermal design is much more integrated while the measuring principle is still the same. The CompaCT scanner [21] was successfully applied for gas-liquid flow investigations [22, 23, 24].

Beside the thermal design, the energy discrimination stage is one of the essential parts to provide high measuring accuracies of both gamma ray CT scanners. This is very important if the radiation flux is highly attenuated. Especially for the usage of ${ }^{137} \mathrm{Cs}$ with an energy line of about $662 \mathrm{keV}$, the energy resolution of the scintillation detectors must be good enough to sufficiently mask the photo effect interaction area in the measured pulse height spectrum. In fact, the energy discrimination stage can be interpreted as a simplified but programmable single channel analyzer (SCA). As a matter of fact, gamma photons do not always deposit their full energy into the detector, but only part of it due to Compton interaction probability. Consequence of it is a loss of gamma photons to be registered and, thus, a longer scanning interval to achieve acceptable gamma photon statistics. Preliminary investigations by Hampel et al. [9] have already revealed that a significant part of the Compton area in the measured spectrum can also be marked as valid for counting if a sufficient detector collimation is arranged. In addition, if more than a single gamma photon energy is applied in a gamma ray CT scanner, as presented by, e.g., [13] or [11], a single energy discrimination stage avoids a simultaneous CT scan of multiple masked energy areas. Thus, it is desirable to measure not only a single photon energy range but a multi energy spectrum of the detected gamma photons.

For this purpose, multi-channel analysers (MCA) are typically applied using peak-sensing or spectroscopic analogueto-digital converters (ADCs) to digitize the amplitude, i.e. the maximal height, of each pulse [25]. The electronics of commercially available MCAs must operate very precisely to achieve a very fine pulse height resolution to be able to resolve multiple photon energy lines with small distances. Unfortunately, this requires elaborated additional electronic devices with additional power consumption. While this is not an issue for a single detector channel, it is challenging for tomography systems with hundreds or thousands of detector channels. Furthermore, as lower spectral resolution is required for scintillation detectors in tomographic applications, a precise peak-sensing electronics is not really required.

Therefore, we present a further development of the modular signal processing board (MSPB) electronics [26], where the single pulse height discrimination stage of each detector channel is upgraded by a novel pulse duration time measurement design. The logic circuit of the existing complex programmable logic device (CPLD) is changed while the residual electronics is left as it is. With the knowledge of the analogue pulse shape, the pulse height spectrum is extracted from the pulse duration time spectrum and vice versa. We present a sophisticated read-out interface that can easily be implemented in CPLDs. The read-out procedure, the measuring accuracy as well as the electronics dead time is analyzed and discussed.

\section{Materials and Methods}

\subsection{The Detector Module of the CompaCT}

The hardware of the entire gamma ray CompaCT scanner is fully described elsewhere [21]. We limit the description here to the detector and its electronics. The detector arc comprises 112 seamlessly arranged scintillation detectors, each composed of lutetium yttrium orthosilicate (LYSO) crystal, $2 \mathrm{~mm}$ wide and $4 \mathrm{~mm}$ high, coupled to an avalanche photo diode (APD). The detector interaction length is about $24 \mathrm{~mm}$ and provides an interaction efficiency of $75 \%$ for gamma photons of $662 \mathrm{keV}$ energy. Compared to sodium iodide $\mathrm{NaI}(\mathrm{TI})$ scintillation material, that in general is exclusively used for gamma spectrometry, the selected LYSO offers a two-times higher stopping efficiency, a six-times faster scintillation light decay time and a non-hygroscopic nature [27]. Thus, detector modules can be assembled more compact, signal pile-ups can be suppressed much better and system dead times can be reduced significantly by using properly dimensioned amplification stages.

The drawback of this small $2 \times 4 \mathrm{~mm}^{2}$ detection crosssection is a worse ratio of photo effect to Compton effect for gamma photons with high energy. As illustrated in Figure 1a, only gamma photons hitting the cross-section orthogonally and in the very center of the scintillation volume are able to deposit their full energy in the LYSO cube. Thus, a certain subset of non-scattered gamma photons will not deposit their full energy into the LYSO cube which causes a deformation of the detected ${ }^{137} \mathrm{Cs}$ spectrum as shown in Figure $1 \mathrm{~b}$ and a reduction of measured non-scattered gamma photons.

The generated charge at each APD is converted into a voltage pulse by a charge sensitive preamplifier stage followed by a pulse shaping stage to provide an almost symmetrical voltage pulse with a duration time of maximal $2 \mu \mathrm{s}$. The amplitude of each pulse is directly proportional to the deposited energy of the gamma photon. Thus, the pulse height spectrum can be interpreted as a gamma photon energy spectrum. The corresponding analogue electronics is modularly designed in full-parallel for all eight detector channels. As mentioned, the CompaCT electronics provides simplified but programmable SCA stages for 
each detector channel as sketched in Figure 2. Here, the analogue voltage pulses are initially connected to a freely programmable dual comparator stage fed with a lower and upper threshold value that define the boundaries of the voltage window in which pulses are valid for counting (see Figure 3).

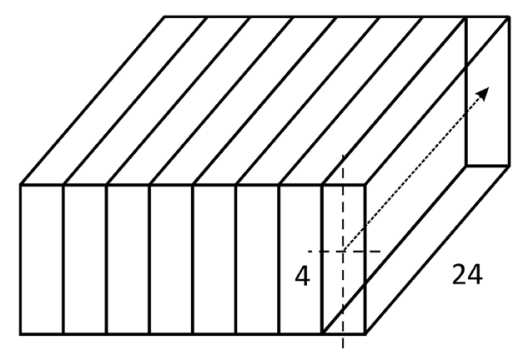

a)

2

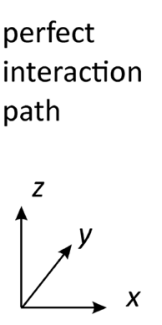

b)

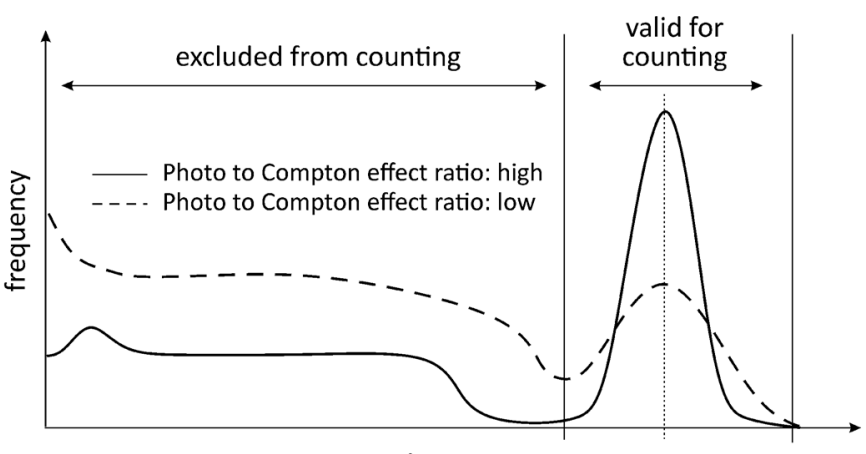

pulse height / gamma photon energy

Figure 1 a Sketch of an eight channel LYSO detector block and $\mathbf{b}$ fictitious spectra of ${ }^{137}$ Cs for different Photo to Compton effect ratios.

Figure 2 Schematic of the modular signal processing board that is designed with single energy area discrimination stages [21].
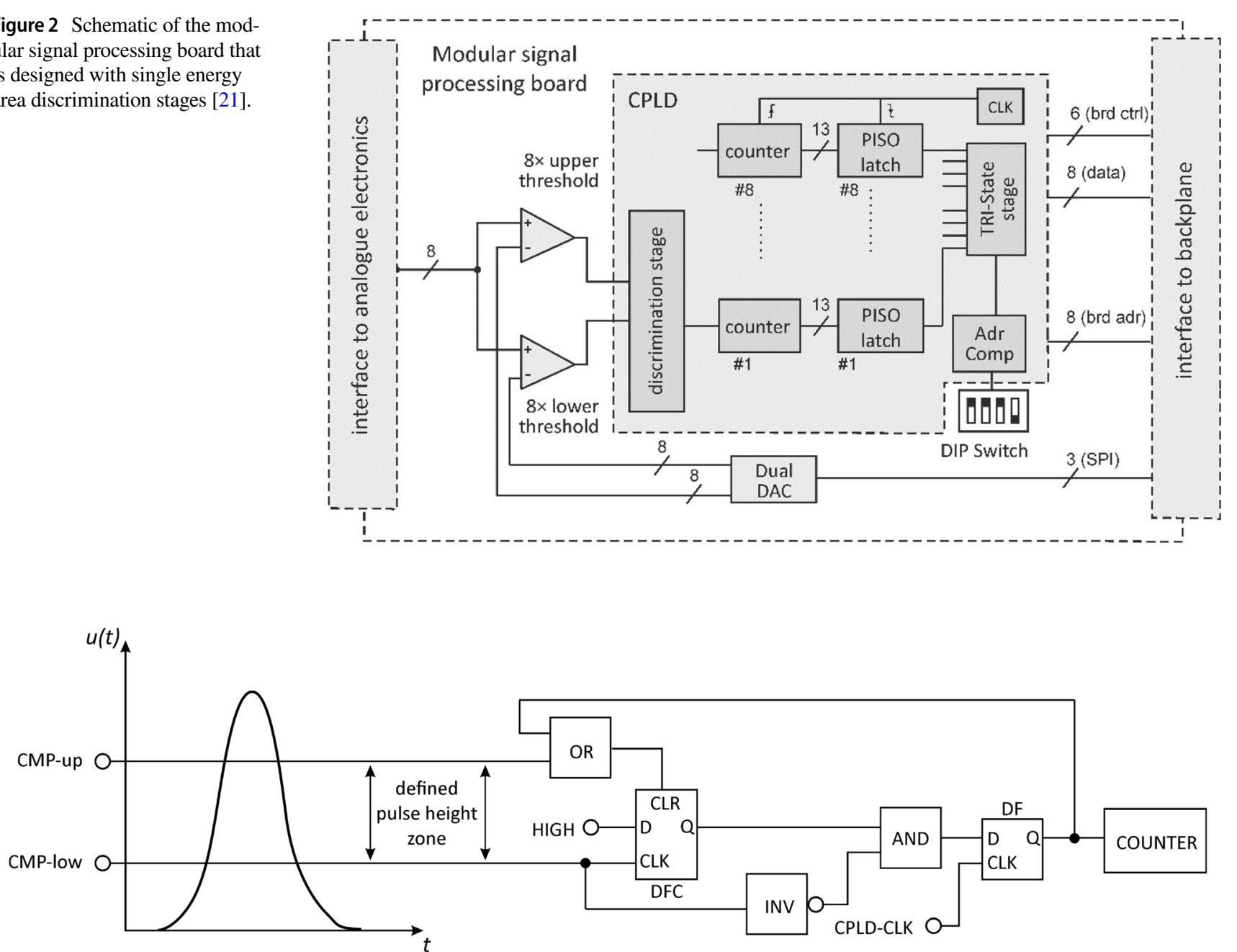

Figure 3 Simplified schematic of the pulse height evaluation stage for a single detector channel. 
The output signals of each comparator pair are connected to a complex programmable logic device containing a logic circuit for pulse height evaluation and counting. As a signal from the lower threshold comparator (CMP-low) sets an edge-triggered flip-flop (DFC) to logical HIGH, this information is stored as long as it is not actively cleared. This can only be achieved in two cases: In the first case, the signal from the upper comparator (CMP-up) is set to logical HIGH, i.e. the analogue signal exceeds the defined pulse height zone and so the pulse is not valid for counting. In the second case, the signal from the lower comparator is cleared and, thus, the subsequent AND stage becomes logical HIGH. Afterwards, another clocked flip-flop device (DF) guides the information for exactly one CPLD-CLK cycle to a subsequent counter and at the same time the feedback loop clears the edge-triggered flip-flop. The counter is increase by one and, thus, a valid event is registered. In this way, valid asynchronous detector signals are synchronously clocked into the CPLD. As illustrated in Figure 2, each detector channel comprises a counter with a value depth of 13 bit followed by a parallel-in-serial-out (PISO) latch that stores the entire counter value within a single CPLD clock. Thus, if the MSPB is addressed, the stored counter values of all eight detector channels can serially be transferred with the least significant bit (LSB) at first using one bit of the 8 bit data bus, respectively.

Note, the unusual bit depth of each counter is due to the limited resources of the used CPLD type "ispMACH4256Ze" (Lattice Semiconductor). As the counters are realized by flip-flop elements only, the 13 bit counter size means the maximal possible bit depth that still allows the routing of the internal logic circuit with acceptable signal speed. However, for the application as programmable SCA that counter size is sufficient.

The compact setup and the low amount of logical devices of such a simple programmable SCA allows for the application in detector systems with thousands of channels. But as basis for simplified MCA purposes this concept cannot be used, as additional threshold voltage stages would have been simultaneously provided which is very complex, a waste of space and electronic power and not economic. Instead, an upgradeable solution for the available modular signal processing board infrastructure is requested that uses the eight dual comparator input signals as well as the eight data output and the six control pins that are already connected to the controller board via a flexible back plane (see Figure 2). In summary, a new logic circuit for the CPLD is requested providing the following two main tasks:

- parallel pulse height spectrum acquisition for each detector channel whereat the pulse amplitude area as well as the number and the width of the pulse height channels are selectable
- intelligent data readout with low (or at best no) gamma photon detection dead time

Furthermore, the counter depth for each pulse height channel must be selectable and a dedicated data readout of specific spectrum areas, i.e. selected gamma photon energy areas, must be realised to optimize the data read-out sequence. However, the quality of the spectral resolution is less important.

\subsection{Concept of Pulse Duration Time Measurement}

To realise a smart multi-channel analyser for tomography application, the approach of pulse duration time (PDT) measurement is chosen. As sketched in Figure 4, there is a relation between the pulse duration time and its amplitude. Assuming two analogue pulses with different amplitudes $\mathrm{U}_{1}$ and $\mathrm{U}_{2}$ the corresponding duration times $\mathrm{T}_{1}$ and $\mathrm{T}_{2}$ at a defined threshold voltage $U_{\text {Trig }}$ can easily be measured using a fast sampling clock. The faster the sampling clock $f_{\text {samp }}$ the more accurate the pulse duration time

$T_{\text {pulse }}=N_{\text {samp }} / f_{\text {samp }}$

can be determined simply by counting the amount of full clock cycles $N_{\text {samp }}$ that fit into the pulse duration. Only an external start/stop trigger voltage $U_{\text {trig }}$ value is required. As applied in standard MCAs, the determined pulse duration time values are used as channel address pointers to acquire, this time, a so-called pulse duration time spectrum. By knowing the pulse shape or by applying a suitable calibration procedure the corresponding pulse height spectrum can finally be deduced.

The advantage of this method is that this logic circuit can easily be implemented to CPLDs or FPGAs (field programmable gate arrays). All required components are available, such as high clocking frequencies, counters and storages. The main drawback of this approach is that in reality the pulses are only roughly Gauss-shaped, but never perfectly symmetric. Furthermore, the relation between pulse duration time and pulse amplitude is not linear. As this is different to standard MCAs, where the pulse height channel width $\Delta u$ is constant over the entire analogue signal acquisition range, the resulting pulse amplitude channel width from a pulse duration MCA needs to be resorted prior to further evaluation.

The relation between the pulse amplitude and duration time can be theoretically analysed or practically measured with test pulses applied at the beginning of the charge sensitive preamplifier stage. For theoretical analysis the voltage pulses are assumed to be Gaussian, e.g. with a mean value $\mu$, a standard deviation of $\sigma$ and an amplitude scalar 
Figure 4 Functional principle of pulse duration time measurement.

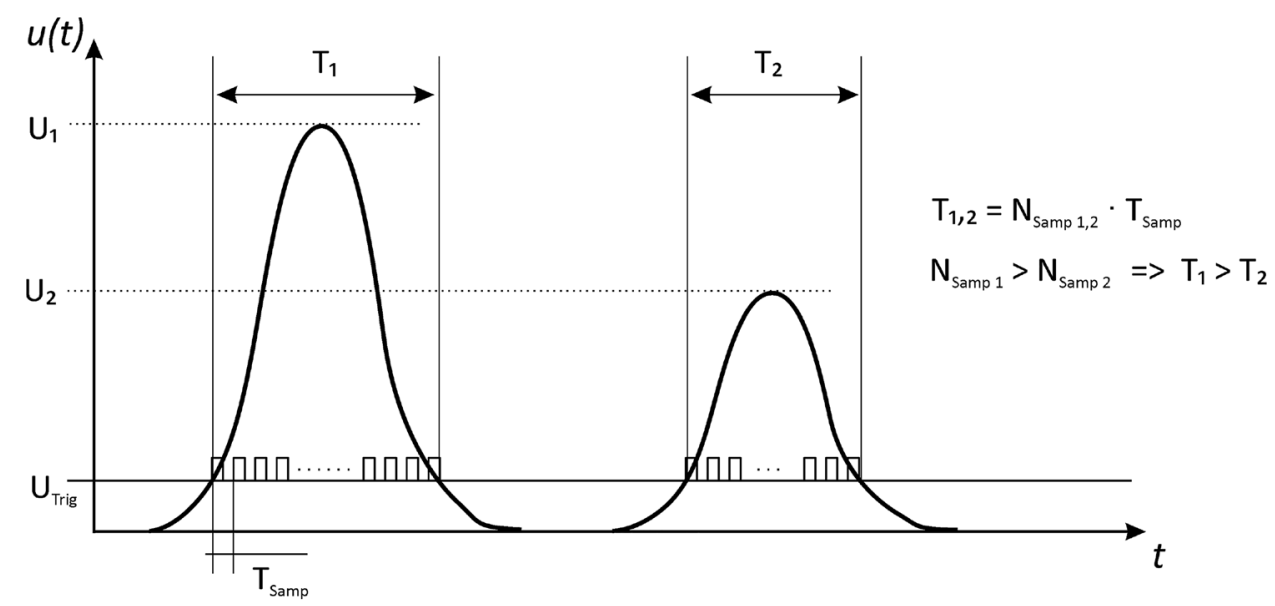

factor of $A$. Thus, pulses with the shapes that are shown in Figure 5a can analytically be described by

$u(t)=\frac{A}{\sqrt{2 \pi \sigma^{2}}} \cdot \mathrm{e}^{\frac{(t-\mu)^{2}}{2 \sigma^{2}}}$

By varying the scalar factor $A$ and applying $\mu=0$ s, pulses with different amplitudes

$u(0)=U_{\max }=\frac{A}{\sqrt{2 \pi \sigma^{2}}}$

can be simulated using Eq. (2) at a constant time point, e.g. $t=0 s$. As can be seen in Figure $5 \mathrm{~b}$ different trigger threshold values $U_{\text {trig }}$ will lead to different pulse duration times $t$ for pulses with the same amplitude. Thus, the applied trigger voltage value $u(t)=U_{\text {trig }}$ must also be considered for pulse height evaluation and, furthermore, plays another key role for the resolution of the pulse height spectrum. Using Eqs. (2) and (3) the pulse height, i.e. its amplitude,

$U=U_{\text {trig }} / e^{-\frac{t^{2}}{2 \sigma^{2}}}$

can be calculated using the selected trigger threshold value, the measured pulse duration time $t$ and the standard deviation $\sigma$ that describes the shape of the analogue pulse.

Eventually, the pulse duration times are discretely measured in constant time intervals $t_{i}$ within the limits of $0<i \leq N_{\text {samp }}$. This leads to a limited but constant channel
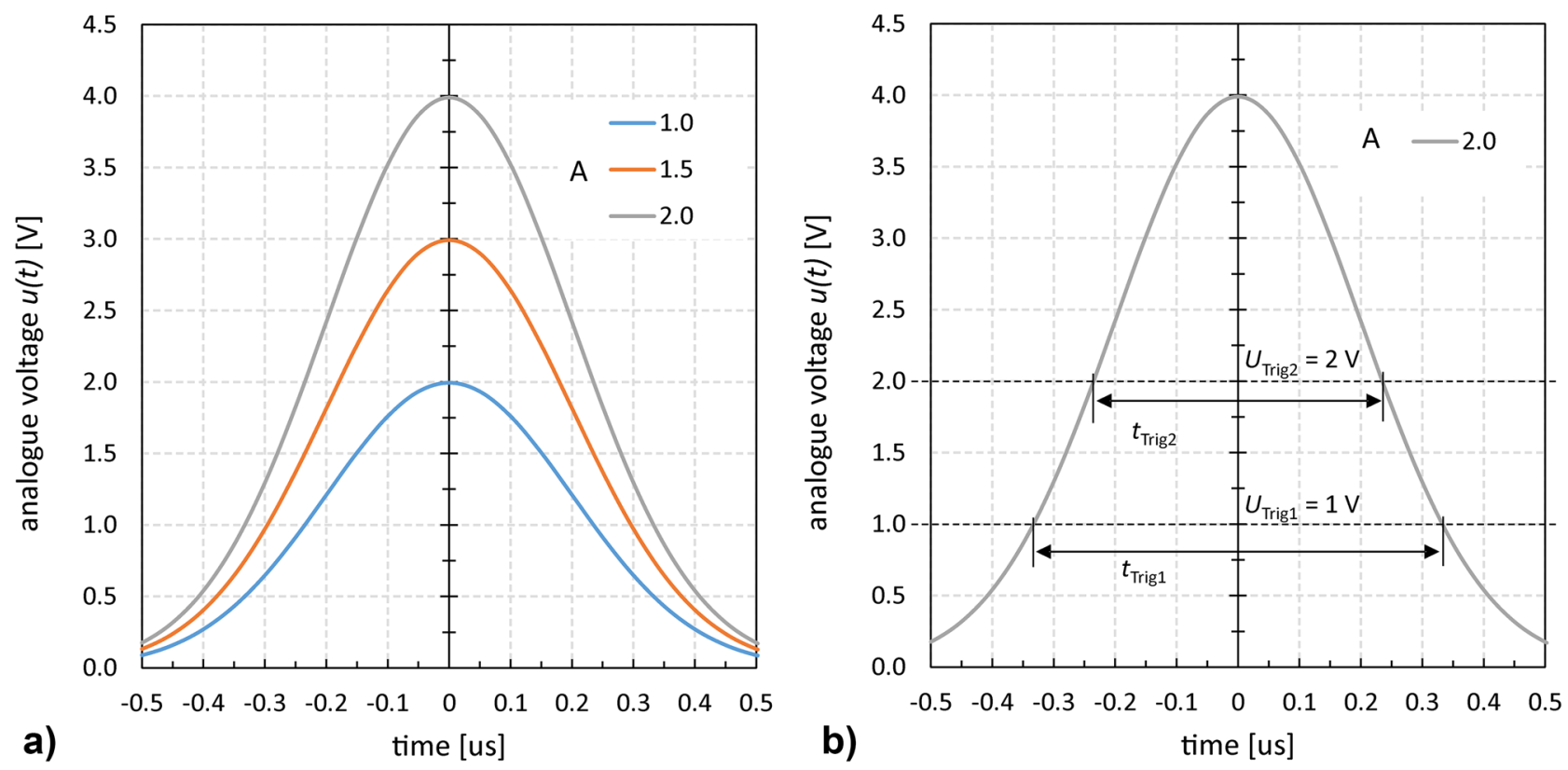

Figure 5 Gaussian-shaped pulses for different scalar factors $A, \mu=0$ s and $\sigma=0.2$ s to described analogue pulses from a detector channel. 
resolution $\Delta t$ for the acquired pulse duration time spectrum $T_{i}$. Thus, the normalization of each element $T_{i}$ to $\Delta t_{i}$ will not affect the shape of the spectrum. The channel resolution can be determined by selecting a proper sampling frequency $f_{\text {samp }}$. But, the correspondingly calculated discrete amplitudes $U_{i}$ from $t_{i}$ using Eq. (4) are not equidistant because of the non-linear correlation between time and amplitude of Gaussian-pulses. That means, the raw data of the recalculated pulse height spectrum $U_{i}$ offers a varying channel resolution $\Delta u_{i}=U_{i+1}-U_{i}$. This time the normalization of each element $U_{i}$ to $\Delta u_{i}$ affects the spectrum shape significantly. Furthermore, the various channel widths $\Delta u_{i}$ must be corrected by stretching the $U_{i}$ spectrum correspondingly. Afterwards, the so obtained pulse height spectrum $U_{i}$ from the time duration spectrum $T_{i}$ can be compared with the results of standard MCAs.

\subsection{Workflow for Pulse Duration Time Measurement}

The implementation of the pulse duration time measurement in a CPLD is rather simple. A precise external or internal sampling clock $f_{\text {samp }}$ must be provided that is faster than the duration of the analogue pulse to be measured. As can be seen in Figure 6a, only three D-FlipFlops and a combined COUNTER/LATCH stage are required to perform pulse duration measurements. As a HIGH signal is connected to DF_COUNT the sampling clock CLK is passed thought the subsequently connected COUNTER. Both, DF_SAVE and $D F \_C L R$ are in CLEAR mode. After DF_COUNT is fed with a LOW signal, the COUNTER stops counting and with the next clock cycle the counter value is stored into a suitable LATCH. After another clock cycle, COUNTER is cleared (see Figure $6 \mathrm{~b}$ ). The pulse duration time is now available for further processing while the COUNTER is ready for the next measurement.

As described above, the currently used CPLD type "ispMACH4256Ze" comprises enough logical devices to realise eight of such PDT stages. Furthermore, it provides also a sufficiently fast internal sampling clock. However, contrary to SCA measuring mode in MCA measuring mode the detected pulse duration times in the COUNTER/LATCH stage must temporarily be registered in sufficiently large register banks during a defined measuring interval. As the available resources of the "ispMACH4256Ze" are almost exhausted with the new eight PDT stages either an external memory must be connected to the current CPLD or a different CPLD with more logical devices must be selected, preferable with the same PIN configuration as the current one. First option is excluded as a complete new board routing design would be necessary.

Thus, the CPLD type "MachXO2" series from LATTICE is selected as those CPLDs provide embedded memory blocks with a block size of 9 kbits and requires only marginal changes in the board design. Furthermore, this series of CPLD provides an internal clock of $88.67 \mathrm{MHz}$ that can easily be adapted using either an internally provided phaselocked loop (PLL) to increase the clock of up to $260 \mathrm{MHz}$ or an additional clock divider to decrease the clock. Thus, the sampling clock can easily be adapted to pulses of different shape. To acquire an entire PDT spectrum, a register bank, i.e. a storage area, with $N_{\text {samp }}$ elements must be provided. Stored COUNTER values are then used as an address pointer to each element of the register bank. When the duration of an analogue pulse was acquired, the value of the addressed storage element is loaded, increased by one and finally written back into the same storage element. The required control sequence is simply attached to the presented logic circuit in Figure 6a.

As analogue pulses from the MSPB are almost symmetrically shaped with a duration time of $1-2 \mu \mathrm{s}$, the number of register elements can be defined to be $N_{\text {samp }}=256=2^{8}$. Fortunately, the "MachXO2-2000HC" provides in total eight embedded memory blocks with a size of 9 kbits each. Thus, separately addressable double-buffers with a maximal size of 1024 bytes $(\sim 8 \mathrm{kbit})$ each, i.e. in total 512 elements with an element size of 2 bytes, can be reserved for each detector
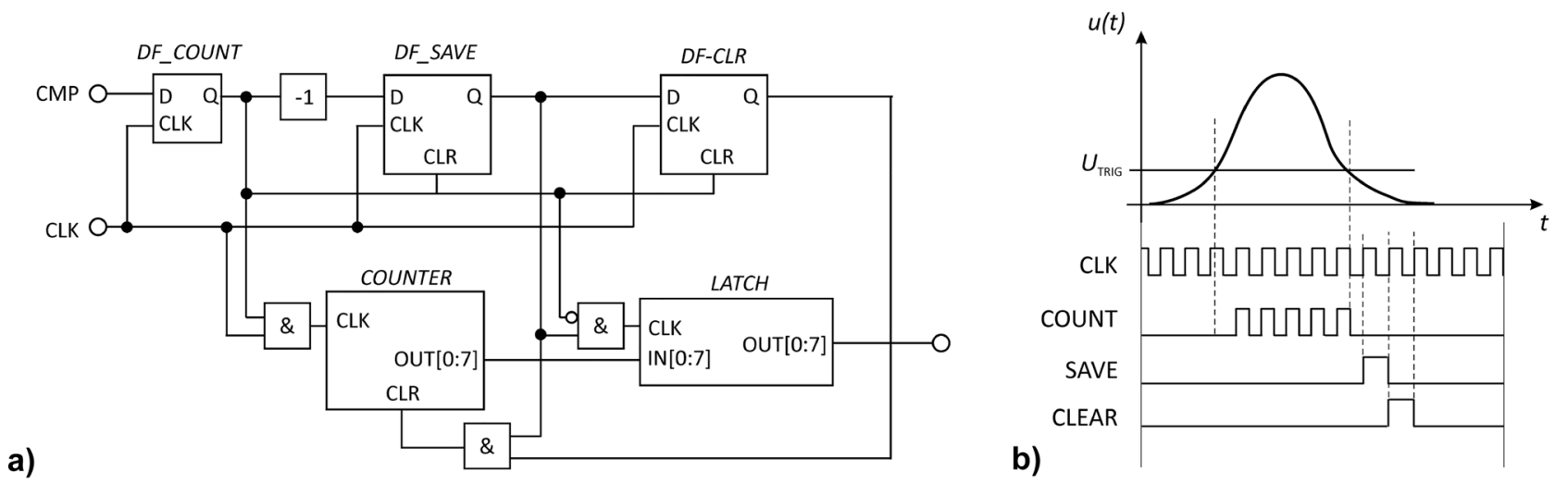

Figure 6 a Logic circuit and $\mathbf{b}$ corresponding signal processing for pulse duration measurement using a discrete and fast sampling clock (CLK). 
channel. Each double-buffer is designed with a write and a read address port of 8 bit plus an additional block selection pin (RAMSFT) that complementary decides which block of 256 elements is currently used for reading and/or writing (see Figure 7).

Note, the selected RAM block size and its structure, i.e. the number and the size of each register element, must be chosen carefully with respect to the maximal pulse duration time that should to be analysed in combination with the applied sampling clock. In case the register element size is too small, e.g. for high gamma photon fluxes or long scanning intervals, the signal acquisition range and the number of register elements can be reduced while the size of the elements are increased correspondingly. This, of course, reduces the pulse height resolution but as mentioned before, no classic MCA is required but a simple one.

In Table 1 the number of control pins are listed that are required to have full control over PDT spectra read-out procedure. Via a microcontroller program, either the entire spectrum or only parts of it, i.e. defined pulse width areas, can be addressed to the 8-bit RAM block output that is, eventually, connected to a parallel-in to serial-out latch to transfer the register data into a serial data stream, i.e. though one bit of the eight bit data bus of the MSPB (see Figure 7). Obviously, the number of required control pins exceeds the number of pins the MSPB provides. Thus, a sophisticated logic circuit is developed and presented in the result part.

\section{Results}

\subsection{Pulse Duration Time Measurement Implementation}

For the implementation of channel-wise PDT measurement within the CPLD type "MachXO2-2000" a logic circuit as illustrated in Figure 8 is designed for each detector channel. The heart is composed of a dual-port RAM block that provides simultaneous access for writing to and reading
Table 1 Required number of (CPLD) pins to provide full control of RAM block data read-out.

\begin{tabular}{ll}
\hline & Number of pins \\
\hline RAM address & $8+1$ \\
RAM read-out & 2 \\
PISO-latch & 2 \\
MSPB address & 8 \\
Total & 21 (available: 14) \\
\hline
\end{tabular}

from the higher or lower RAM block exclusively. In detail, a double-buffer with 256 elements each is provided with a single element size of 2 byte. Each element can be addressed by the Address decoder. As pulse duration sampling clock the internal CPLD clock of $88.67 \mathrm{MHz}$ is accelerated by a factor of three by the PLL to provide a frequency of approx. $266 \mathrm{MHz}$. While the RAM element addressing for data acquisition from the detector is directly provided by the LATCH as described above, the data readout electronics is now of interest. As only six control lines are available at the MSPB backplane, a combined data read-out logic circuit is developed.

In Figure 8 (highlighted in green), the combined RAM addressing and data read-out clocking solution is given. As the correct RAM block is selected for data read-out by the RAMSFT control line, the start address of a coherent 16 element block is selected using an external four bit address (RAMADR) as high nibble, i.e. bit 4 to bit 7 , for the internal RAM block address. The lower nibble is provided by an 8-bit COUNTER that is fed with the data clocking signal (RAMCLK). As can be seen in Figure 8, after 16 clocks (RAMCLK) the lower nibble of the 16 element block address is internally increased by one. The 16 clocks are used as bit address decoder to connect the 16 bit element value to one of the available eight data pins of the MSPB serially with the LSB first. With the last clock (the $16^{\text {th }}$ clock) the content of the addressed element is finally erased. After full or partial read-out of all 16

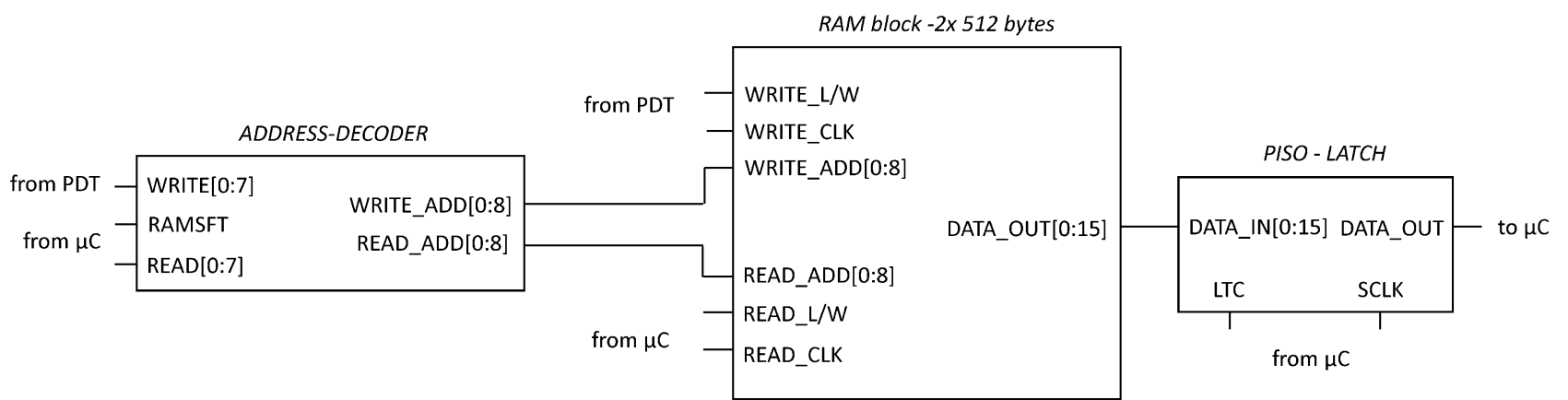

Figure 7 Schematic of data double buffering to store and load PDT spectra from a defined RAM block simultaneously. 


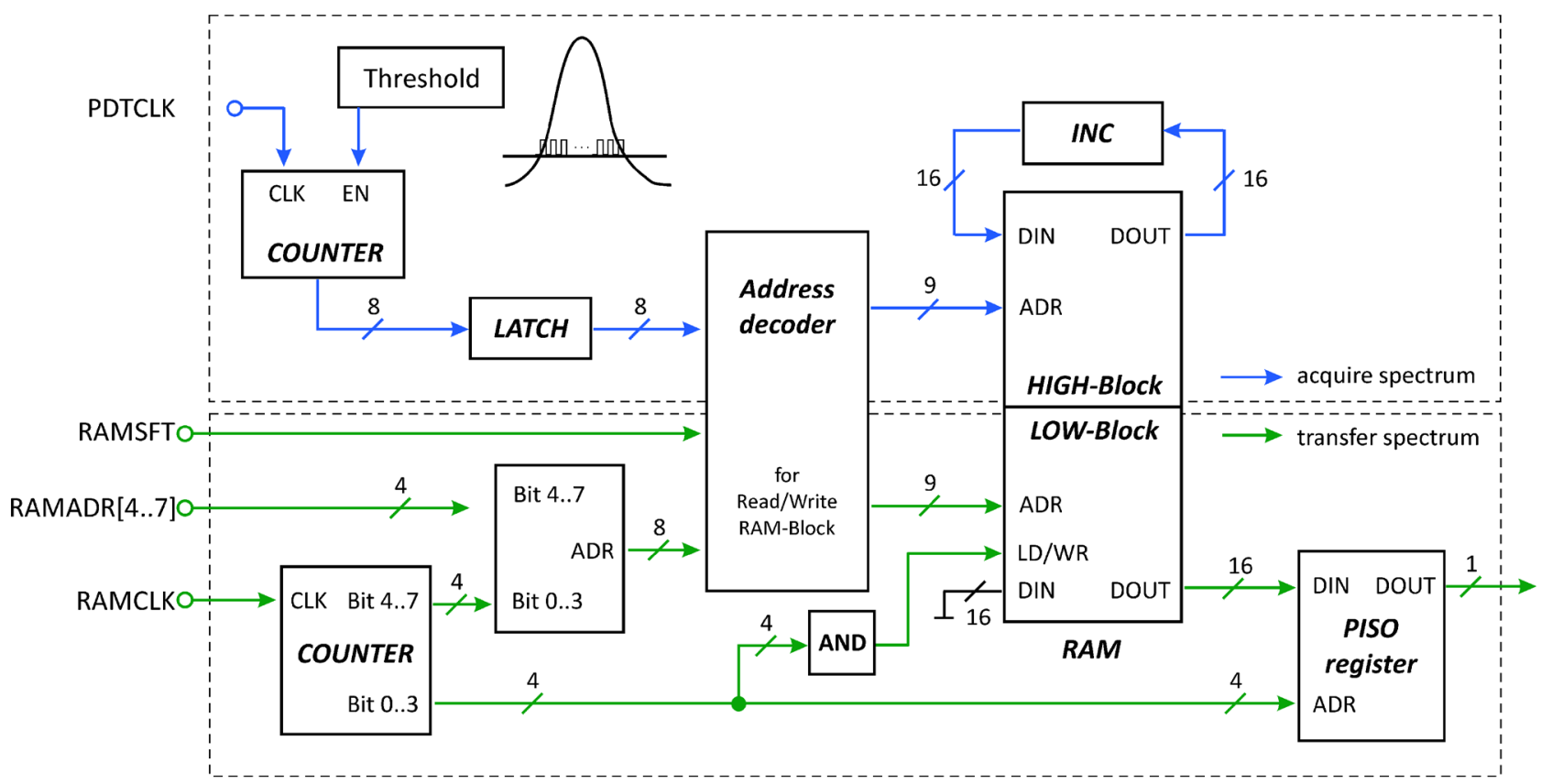

Figure 8 Schematic of logic circuit for acquiring and transferring a pulse duration time spectrum of a single detector channel.

blocks, writing and reading blocks are swapped using the RAMSFT control line.

Thus, a seamless gamma photon evaluation and corresponding pulse duration time spectrum transfer is achieved. The effective system dead time, i.e. the time in which a gamma photon cannot be registered, of the detector module

$t_{\text {dead }}=6 / f_{\text {samp }}$

is only defined by the pulse duration time measurement logic itself to be six sample clock cycles which means that the dead time of the system depends on the selected sampling frequency. However, as $f_{\text {samp }}$ must always significantly be higher than the pulse duration time $t_{i}$, the system dead time can always be assumed to be negligible.

\subsection{Pulse Height Resolution Analysis}

In the next step, the usage of Gauss-shaped pulses to analytically describe the function of realistic pulses is evaluated by comparing the pulse duration times of both. Therefore, the analogue circuit of the MSPB is designed in a simulation tool and test signals are provided that lead to the pulses shown in Figure 9a with defined amplitudes between 1 and 5 V. As can be seen in Figure 9b, the pulse duration times of realistic pulses can also be described very well with an empirically optimized Gaussian function, as is shown in Figure 9c. The optimized Gaussian function is realised with a standard deviation value of $\sigma=0.33 \mu s$ and evaluated with three different scalar factors $A$, as specified in Eq. (2). It can be observed, that the duration time accuracy (Figure 9b) is sufficiently predicted for tomography application.

Now, the resolution of the pulse amplitude can be analysed using the identified Gaussian function. As described above, the CPLD provides an internal basis clock of approx. $f_{\text {samp }}=266 \mathrm{MHz}$ and each detector channel is supported with a block register of $n=256$ elements to acquire the pulse duration time spectrum. Thus, pulse duration times of maximal

$t_{\max }=\frac{256}{266 \mathrm{MHz}}=962 \mathrm{~ns}$

can be acquired with this configuration with discrete time steps of $t_{i}=3.76 \mathrm{~ns}$. Unfortunately, the duration time of pulses from the MSPB may be longer than $t_{\max }$ for pulses with high amplitudes and low trigger voltages (see Figure 9b). Thus, either the analogue detector signal acquisition range is limited at the beginning or the end, i.e. the trigger voltage value must be set carefully, or the measurement accuracy of the pulse duration time is decreased by subdividing the sampling clock by a factor of $2^{\mathrm{n}}$. The decision depends on the purpose: either mapping the spectrum as good as possible or achieving/setting the signal range as large as possible.

Note, also a restructured block register with more elements but less storage size per element would solve the problem, but requires a correspondingly modified logic circuit that needs to be loaded within the CPLD.

For the presentation of an exemplary performance analysis, we decide here to subdivide the basis clock by $2^{1}=2$, i.e. 

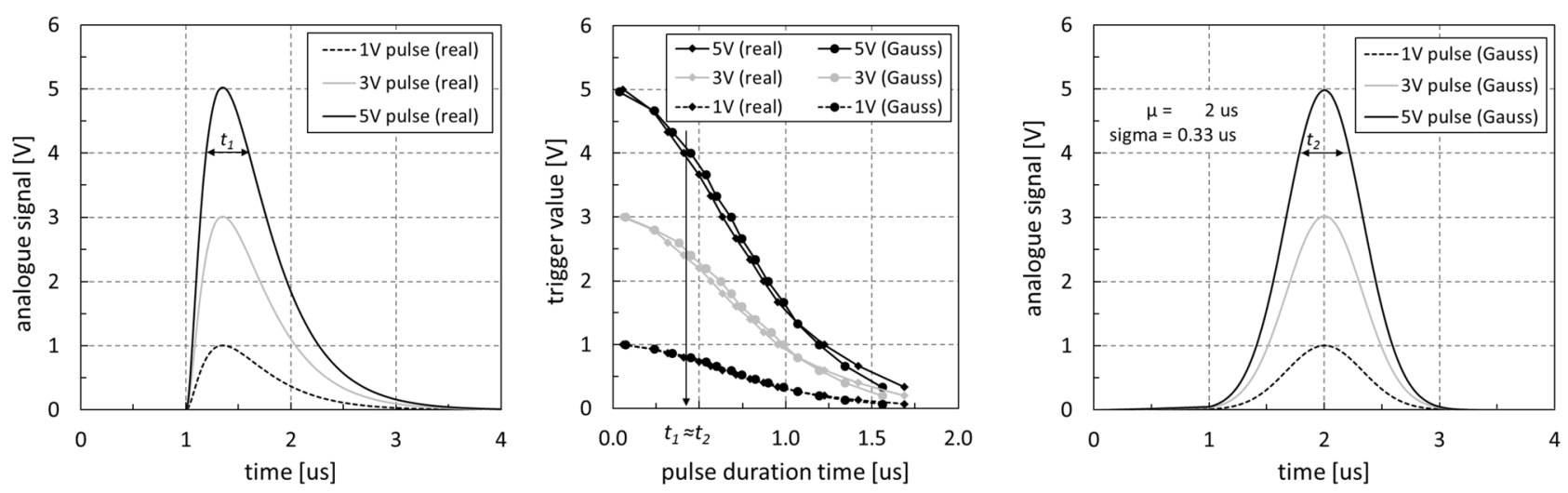

Figure 9 Time signal of a simulated and $\mathbf{c}$ perfectly Gaussian-shaped voltage pulses with $1 \mathrm{~V}, 3 \mathrm{~V}$ and $5 \mathrm{~V}$ amplitude and $\mathbf{b}$ corresponding pulse duration times for various trigger values.

pulse durations with up to $1.94 \mu$ s can securely be evaluated. To investigate the amplitude sensitivity $\Delta u_{i}$ from discretely measured time stamps $t_{i}=i \cdot \frac{1}{133} \mathrm{MHz}=i \cdot 7.5 \mathrm{~ns}$, Eq. (4) is applied and the amplitude differences $\Delta u_{i}$ of subsequent time steps $t_{i}$ are calculated. In Figure 10 the resulting sensitivity of pulse height resolution is plotted for three different trigger values $U_{\text {trig }}$. As can be seen, the closer the trigger value is set to the area of interest, i.e. the photo peak zone of ${ }^{137} \mathrm{Cs}$, the more accurate the signal amplitudes can be calculated from the duration time measurement. Additionally, the quality of the signal amplitude resolution

$R_{i}=\frac{\Delta u_{i}}{\left(U_{i}+U_{i+1}\right) / 2}=\frac{U_{i}-U_{i+1}}{\left(U_{i}+U_{i+1}\right) / 2}$

can be quantified. As illustrated at the top of Figure 10, the quality of scintillation detectors for tomographic applications is about $14 \%$ for full width at half maximum (FWHM). Thus, the minimal required quality of amplitude resolution $R$ can be deviated to be able to reconstruct significant characteristics within the pulse height spectrum.

\subsection{Spectra measurements of ${ }^{137} \mathrm{Cs}$}

Finally, pulse duration time spectra are acquired with the presented CPLD logic circuit design. As mono-energetic isotopic source ${ }^{137} \mathrm{Cs}$ with an activity of about $28 \mathrm{kBq}$ is used. A large CdZnTe detector CZT/500S (Ritec) is selected that is operated with the preamplifier PA-101C (Ritec). In fact, its spectral resolution of about $7 \%$ is significantly better compared to scintillation detectors. But the spectral characteristics, such as photo peak, Compton edge and/or backscattered peak, are much better visible and, thus, more illustrative to prove the proposed measuring technique of PDT. To determine best sampling frequency and trigger value, classic pulse height spectra are initially acquired and corresponding pulse duration time spectra are artificially calculated for various $f_{\text {samp }}$ and $U_{\text {trig }}$ and a constant number of register elements of $N_{\text {samp }}=256$.

To acquire reliable pulse height spectra, the multichannel analyser MCA-166 from (GBS Elektronik, Germany) is used. This device biases the CdZnTe detector with $U_{\text {bias }}=-1400 \mathrm{~V}$ and its internal parameters are selected in a way that a pulse height spectrum with the photo peak at approximately $2.5 \mathrm{~V}$ is obtained. The pulse shaping time is set to $1 \mu$ s, i.e. pulse duration times of up to $6 \mu$ are expected. In a first step, pulse height spectra are recorded with both 256 and 1024 channels for an interval of $300 \mathrm{~s}$. As shown in

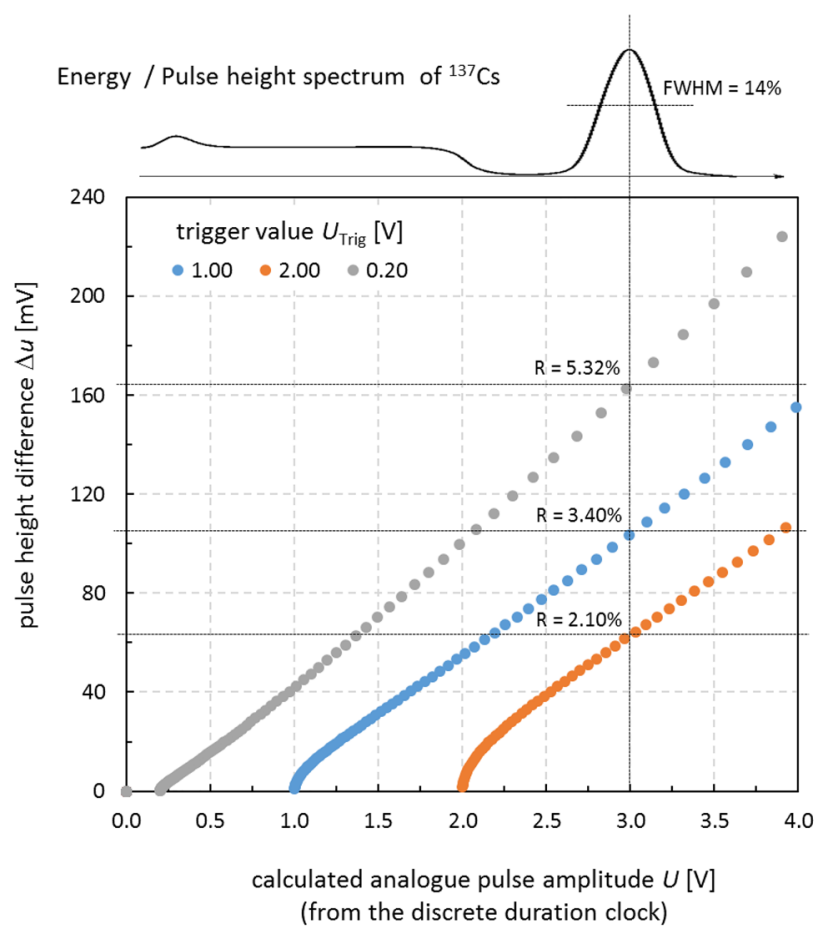

Figure 10 Pulse height resolution map for three threshold values $U_{\text {trig }}$ $=0.2 \mathrm{~V}, 1.0 \mathrm{~V}$ and $2.0 \mathrm{~V}$ using the analogue detector signals from the MSPB and a sampling clock of $133 \mathrm{MHz}$. 
Figure 11 Acquired pulse height spectra of ${ }^{137} \mathrm{Cs}$ using a large CZT detector and the multi-channel analyzer MCA166 with 256 and 1024 channels, respectively.

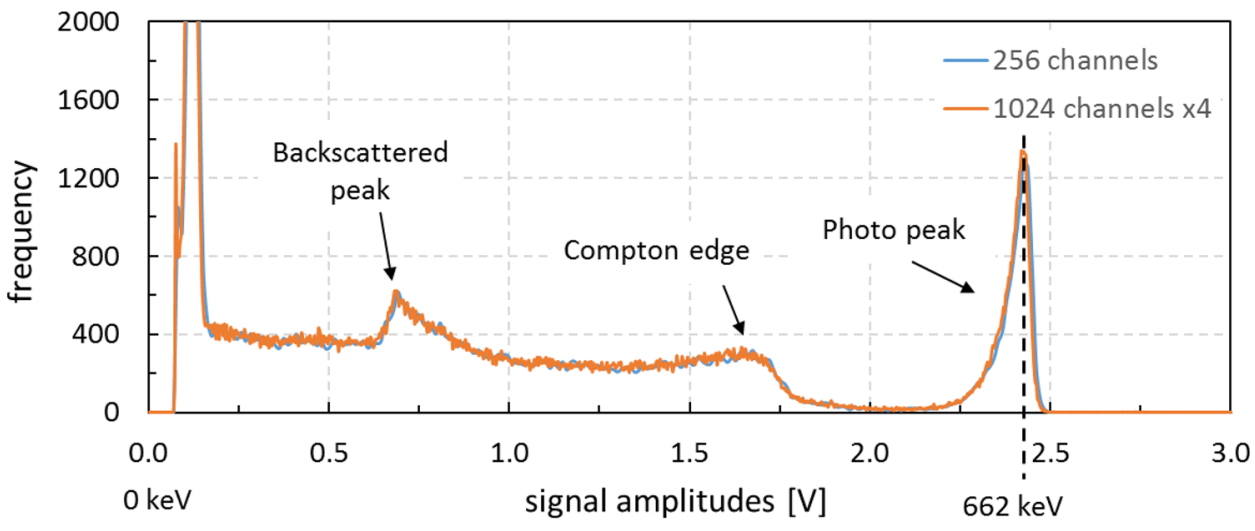

Figure 11, both pulse height spectra map typical characteristics of the ${ }^{137} \mathrm{Cs}$ source very well. For better visualisation, the spectrum with 1024 channels is multiplied by the factor $1024 / 256=4$

In a second step, representative analogue pulses (amplitudes: $0.6 \mathrm{~V}, 1.0 \mathrm{~V}$ and $1.5 \mathrm{~V}$ ) from the MCA-166 are analysed correspondingly to Figure 9 to extract the required Gaussian standard deviation value to be $\sigma=1.65 \mu \mathrm{s}$. In Figure 12 representatively calculated pulse duration time curves for different trigger values $U_{\text {trig }}$ are shown.

In a final step, PDT spectra are acquired using $38 \mathrm{MHz}$ as sampling frequency. In Figure 13 artificially calculated and directly measured pulse height spectra are shown for three different trigger values with a voltage difference of $500 \mathrm{mV}$, respectively. The constant voltage offset of $400 \mathrm{mV}$ is due to imperfect photo peak positioning to $2.5 \mathrm{~V}$. It can be seen that the shape of the directly measured pulse height spectra (Figure 13b) perfectly corresponds to the artificially

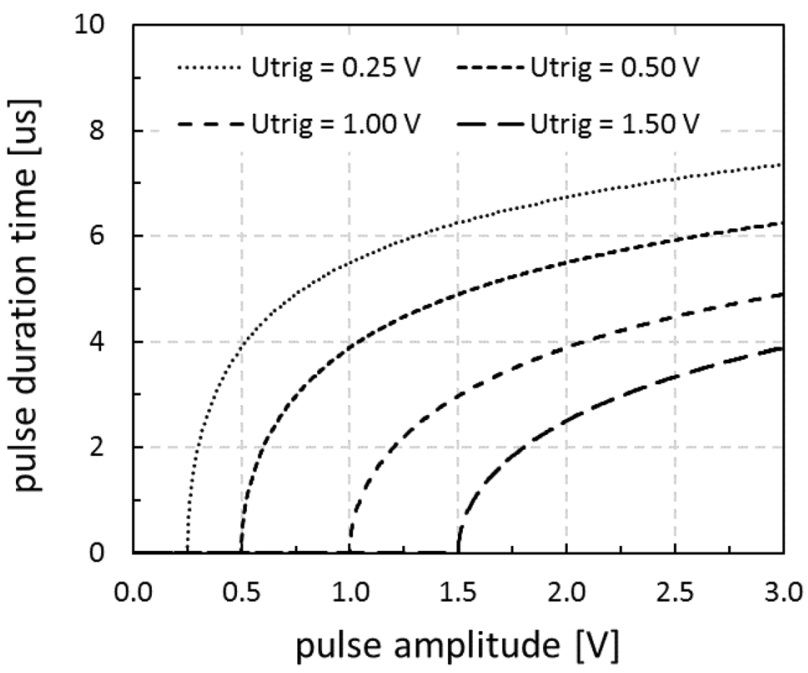

Figure 12 Set of pulse duration time curves for different trigger values and the empirically determined Gaussian function that describes the duration times of analogue pulses from the MCA- 166 . calculated ones (Figure 13a). Especially the ${ }^{137} \mathrm{Cs}$ photo peak as well as the Compton edge are well developed. The distances of the photo peak for different trigger values could also be predicted very well. Furthermore, the widening and the corresponding reduction of the maximum frequency of the photo peak with increasing trigger value $U_{\text {trig }}$ could also be measured and proves, thereby, both the measuring principle as well as the functionality of the theoretical approach to optimize the shape of the PDT spectrum using the Gaussian function as an substitution for analogue pulses.

Interestingly, the backscattered peak is not well developed in the PDT spectrum. As it is too far away from the photo peak area, the resulting pulse amplitude resolution is so fine that the backscattering peak is mapped in the PDT spectra strongly stretched. Eventually, a quantitative comparison of the ${ }^{137} \mathrm{Cs}$ photo peak reveals a marginal worse amplitude resolution in the directly measured pulse height spectra. This is mainly caused by additional noise from the applied trigger value stage and jittering of the sampling frequency $f_{\text {samp }}$ provided by the CPLD.

\section{Summary}

In this paper, an approach for the enhancement of gamma photon counting electronics for an existing gamma ray computed tomography scanner was presented. Instead of defining a single valid area for non-scattered gamma photon counting, a simplified spectral measurement approach is proposed. Therein, each electrical pulse, generated by an interacting gamma photon, is evaluated by its duration time and correspondingly registered in a pre-defined register block, whose elements are directly addressed by the detected pulse duration time.

A corresponding new general logic circuit for an existing tomography electronics is presented and discussed. A dedicated logic circuit was implemented within a CPLD and its performance was successfully evaluated. Finally, pulse duration time spectra of ${ }^{137} \mathrm{Cs}$ were acquired using the CPLD 


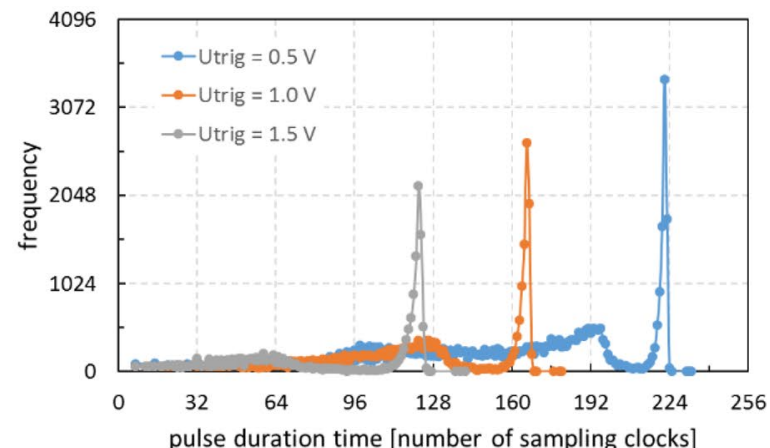

a)

pulse duration time [number of sampling clocks]

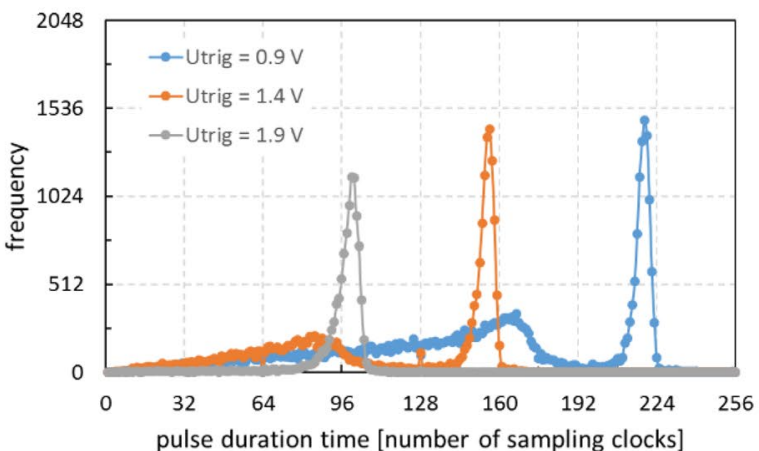

b)

Figure 13 Pulse duration time spectra of ${ }^{137} \mathrm{Cs}$ a calculated from measured pulse height spectra with MCA-166 and b measured with the presented CPLD logic circuit.

logic circuit design. These PDT spectra were compared with PDT spectra that are calculated from pulse height spectra obtained from a commercially available multi-channel analyser. The comparison reveals very good qualitative matches, e.g. with the position and the shape of the photo peak of ${ }^{137} \mathrm{Cs}$.

Thus, the developed logic circuit can easily be used as upgrade for the existing electronics. It enables a new data post-processing and analysing step which will increase the image quality of the tomographic scanner and broaden its applicability to more complex measurement tasks.

Funding Open Access funding enabled and organized by Projekt DEAL. This project was not funded by any project.

Open Access This article is licensed under a Creative Commons Attribution 4.0 International License, which permits use, sharing, adaptation, distribution and reproduction in any medium or format, as long as you give appropriate credit to the original author(s) and the source, provide a link to the Creative Commons licence, and indicate if changes were made. The images or other third party material in this article are included in the article's Creative Commons licence, unless indicated otherwise in a credit line to the material. If material is not included in the article's Creative Commons licence and your intended use is not permitted by statutory regulation or exceeds the permitted use, you will need to obtain permission directly from the copyright holder. To view a copy of this licence, visit http://creativecommons.org/licenses/by/4.0/.

\section{References}

1. Rollbusch, P., Becker, M., Ludwig, M., Bieberle, A., Grünewald, M., Hampel, U., Franke, R. (2015). Experimental investigation of the influence of column scale, gas density and liquid properties on gas holdup in bubble columns. International Journal of Multiphase Flow, 75, 88-106. https://doi.org/10.1016/j.ijmultiphaseflow.2015. 05.009

2. Stannarius, R., Sancho Martinez, D., Boerzsoenyi, T., Bieberle, M., Barthel, F., \& Hampel, U. (2019). High-speed X-ray tomography of silo discharge. New Journal of Physics, 21, 113054. https:// doi.org/10.1088/1367-2630/ab5893
3. Schäfer, T., Neumann-Kipping, M., Bieberle, A., Bieberle, M., \& Hampel, U. (2020). Ultrafast X-ray CT imaging for hydrodynamic investigations of gas-liquid two-phase flow in centrifugal pumps. Journal of Fluids Engineering, 142(4), 041502. https://doi.org/ 10.1115/1.4045497

4. Sohr, J., Bieberle, M., George, G. R., Flechsig, S., Kenig, E. Y., Schubert, M., \& Hampel, U. (2019). Comparative assessment of different image processing methods to determine the gas-liquid interfacial area in froth regimes of sandwich packings from ultrafast X-ray tomography image data. Chemical Engineering Research and Design, 147, 676-688. https://doi.org/10.1016/j. cherd.2019.05.039

5. Neumann-Kipping, M., Bieberle, A., Hampel, U. (2020). Investigations on bubbly two-phase flow in a constricted vertical pipe. International Journal of Multiphase Flow, 130, 103340. https:// doi.org/10.1016/j.ijmultiphaseflow.2020.103340

6. Bieberle, Martina, \& Barthel, Frank. (2016). Combined phase distribution and particle velocity measurement in spout fluidized beds by ultrafast X-ray computed tomography. Chemical Engineering Journal, 285, 218-227. https://doi.org/10.1016/j.cej.2015.10.003

7. Leon, M. A., Maas, R. J., Bieberle, A., Schubert, M., Nijhuis, T. A., van der Schaaf, J., Hampel, U., \& Schouten, J. C. (2013). Hydrodynamics and gas-liquid mass transfer in a horizontal rotating foam stirrer reactor. Chemical Engineering Journal, 217(1), 10-21. https://doi.org/10.1016/j.cej.2012.11.104

8. De Vuono, A. C., Schlosser, P. A., Kulacki, F. A., \& Munschi, P. (1980). Design of an isotopic CT scanner for two phase flow measurements. IEEE Transactions on Nuclear Science, 27, 814-820.

9. Hampel, U., Bieberle, A., Hoppe, D., Kronenberg, J., Schleicher, E., Sühnel, T., Zimmermann, F., \& Zippe, C. (2007). High resolution gamma ray tomography scanner for flow measurement and non-destructive testing applications. Review of Scientific Instruments, 78, 103704. https://doi.org/10.1063/1.2795648

10. Kumar, S. B., \& Dudukovic, M. P. (1997). Computer-assisted gamma and X-ray tomography: Application to multiphase flow (p. 48). Proceeding of Non-invasive Monitoring of Multiphase Flows.

11. Varma, R., Bhusarapu, S., O’Sullivan, J. A., \& Al-Dahhan, M. H. (2007). A comparison of alternating minimization and expectation maximization algorithms for single source gamma ray tomography. Measurement Science \& Technology, 19, 015506. https://doi. org/10.1088/0957-0233/19/1/015506

12. Hoffmann, R., \& Kögl, T. (2017). An ATEX-proof gamma tomography setup for measuring liquid distribution in process equipment. Flow Measurement and Instrumentation, 53, 147-153. https://doi.org/10.1016/j.flowmeasinst.2016.05.009 
13. de Mesquita CH, de Sousa Carvalho DV, Kirita R, Vasquez PA, Hamada MM(2014). Gas-liquid distribution in a bubble column using industrial gamma-ray computed tomography. Radiation Physics and Chemistry, 95, 396-400. https://doi.org/10.1016/j. radphyschem.2013.02.028

14. Johansen, G. A., Frøystein, T., Hjertakery, B. T., \& Olsen, Ø. (1996). A dual sensor flow imaging tomographic system. Measurement Science \& Technology, 7, 297-307.

15. Bieberle, A., Schleicher, E., \& Hampel, U. (2010). Temperature control design for a high-resolution gamma ray tomography detector. Review of Scientific Instruments, 81, 014702.

16. Bieberle, A., Hoppe, D., Schleicher, E., Hampel, U. (2011). Void measurement using high-resolution gamma-ray computed tomography. Nuclear Engineering and Design, 241(6), 2086-2092. https://doi.org/10.1016/j.nucengdes.2011.03.028

17. Visscher, F., Bieberle, A., Schubert, M., van der Schaaf, J., de Croon, M., Hampel, U., \& Schouten, J. (2012). Water and n-heptane volume fractions in a rotor-stator spinning disc reactor. Industrial \& Engineering Chemistry Research, 51, 16670-16676. https://doi. org/10.1021/ie301439s

18. Tschentscher, R., Schubert, M., Bieberle, A., Nijhuis, T. A., van der Schaaf, J., Hampel, U., \& Schouten, J. C. (2013). Gas holdup of rotating foam reactors measured by $\gamma$-tomography - effect of solid foam pore size and liquid viscosity. AIChE Journal, 59(1), 146-154. https://doi.org/10.1002/aic.13787

19. Bieberle, A., Schlottke, J., Spies, A., Schultheiss, G., Kühnel, W., Hampel, U. (2015). Hydrodynamics analysis in micro-channels of a viscous coupling using gamma-ray computed tomography. Flow Measurement and Instrumentation, 45, 288-297. https://doi.org/ 10.1016/j.flowmeasinst.2015.07.008

20. Hampel, U., Hoppe, D., Bieberle, A., Kernchen, R., Diele, K-H. , Schleicher, E., et al. (2008). Measurement of Fluid Distributions in a Rotating Fluid Coupling Using High Resolution Gamma Ray
Tomography. Journal of Fluids Engineering, 130(9). https://doi. org/10.1115/1.2953295

21. Bieberle, A., Nehring, H., Berger, R., Arlit, M., Härting, H.-U., Schubert, M., \& Hampel, U. (2013). Compact high-resolution gamma-ray computed tomography system for multiphase flow studies. Review of Scientific Instruments, 84, 033106. https://doi. org/10.1063/1.4795424

22. Härting, H.-U., Bieberle, A., Lange, R., Larachi, F., \& Schubert, M. (2015). Hydrodynamics of co-current two-phase flow in an inclined rotating tubular fixed bed reactor: Wetting intermittency via periodic catalyst immersion. Chemical Engineering Science, 128, 147-158. https://doi.org/10.1016/j.ces.2015.02.008

23. Timaeus, R., Schleicher, E., Bieberle, A., Hampel, U., \& Schubert, M. (2020). Identification of the process windows of inclined rotating fixed-bed reactors with concentric tube: A hydrodynamic analysis. Chemical Engineering Science, 220, 115637. https://doi.org/10. 1016/j.ces.2020.115637

24. Vishwakarma, V., Schleicher, E., Bieberle, A., Schubert, M., \& Hampel, U. (2021). Advanced flow profiler for two-phase flow imaging on distillation trays. Chemical Engineering Science, 231, 116280. https://doi.org/10.1016/j.ces.2020.116280

25. Knoll, G. F. (2010). Radiation detection and measurement (4th ed.). Wiley.

26. Bieberle, A., Berger, R., Yadav, R., Schleicher, E., \& Hampel, U. (2011). "Design of a modular signal processing board (MSPB) for gamma-ray imaging applications. Nuclear Instruments and Methods in Physics Research A, 663, 14-21. https://doi.org/10. 1016/j.nima.2011.09.060

27. Saint-Gobain (2006) Physical properties of common inorganic scintillators. Technical Information, Crystal Properties.

Publisher's note Springer Nature remains neutral with regard to jurisdictional claims in published maps and institutional affiliations. 